\title{
DIAGNÓSTICOS DE ENFERMAGEM EM PACIENTES ONCOHEMATOLÓGICOS SUBMETIDOS A TRATAMENTO QUIMIOTERÁPICO
}

\author{
NURSING DIAGNOSES IN ONCOHEMATOLOGICAL PATIENTS \\ SUBMITTED TO CHEMOTHERAPEUTIC TREATMENT
}

\section{DIAGNÓSTICOS DE ENFERMERÍA EN PACIENTES \\ ONCOHEMATOLÓGICOS SUBMETIDOS EL TRATAMIENTO QUIMIOTERÁPICO}

\author{
Isadora Braga Calegari ${ }^{1}$, Aldenora Laísa Paiva de Carvalho Cordeiro ${ }^{2}$, Thaís Santos Guerra \\ Stacciarini $^{3}$, Lúcia Aparecida Ferreira ${ }^{4}$
}

\section{RESUMO}

Objetivo: identificar os diagnósticos de Enfermagem mais frequentes em pacientes com doenças oncohematológicas submetidos a tratamento quimioterápico antineoplásico. Método: estudo retrospectivo, documental. Os dados foram coletados de 17 prontuários de pacientes hospitalizados na unidade de oncohematologia de um Hospital Público de Ensino, em março de 2017. Os dados foram analisados por estatística descritiva. Resultados: a maioria dos prontuários era de pacientes do sexo masculino (70,6\%); a média de idade foi de 41,2 anos. Os tipos de cânceres mais frequentes foram linfoma não Hodgkin e leucemia mieloide aguda, com $4(23,6 \%)$ ocorrências. Os diagnósticos de Enfermagem mais frequentes foram: proteção ineficaz e risco de infecção, com 17 (100\%) ocorrências; risco de mucosa oral prejudicada e risco de quedas, com $12(70,6 \%)$ casos. Conclusão: Houve predomínio dos diagnósticos de Enfermagem de proteção ineficaz e risco de infecção, estes estiveram relacionados ao câncer, ao tratamento quimioterápico, à realização de procedimentos invasivos e imunossupressão.

Descritores: Diagnóstico de Enfermagem. Hematologia. Enfermagem Oncológica. Processo de Enfermagem.

\footnotetext{
${ }^{1}$ Enfermeira. Especialista em Saúde do Adulto na modalidade Residência Integrada Multiprofissional em Saúde. Mestranda em atenção à Saúde do Programa de Pós-graduação em Atenção à Saúde da Universidade Federal do Triângulo Mineiro, Uberaba, Minas Gerais, Brasil.

${ }^{2}$ Enfermeira do Serviço de Educação em Enfermagem do Hospital de Clínicas da UFTM (HC/UFTM). Mestre em Atenção à Saúde. Doutoranda em Ciências da Saúde pela Escola de Enfermagem de Ribeirão Preto da Universidade de São Paulo, Ribeirão Preto, SP, Brasil.

${ }^{3}$ Enfermeira. Doutora em ciências da saúde. Enfermeira do Serviço de Educação em Enfermagem do Hospital de Clínicas da Universidade Federal do Triangulo Mineiro, Uberaba, Minas Gerais, Brasil.

${ }^{4}$ Graduada Bacharel em Enfermagem e Obstetrícia. Graduada em Licenciatura em Enfermagem. Mestrado em Enfermagem Psiquiátrica. Doutorado em Enfermagem. Professor Adjunto do Curso de Graduação em Enfermagem da Universidade Federal do Triângulo Mineiro, Uberaba, Minas Gerais, Brasil.
} 


\begin{abstract}
Objective: to identify the most frequent nursing diagnoses in patients with oncohematologic diseases submitted to antineoplastic chemotherapy. Method: retrospective, documentary study. Data were collected from 17 patient charts of patients hospitalized at the oncohematology unit of a Public Teaching Hospital in March 2017. Data were analyzed by descriptive statistics. Results: most of the medical records were male patients $(70.6 \%)$; the mean age was 41.2 years. The most frequent types of cancers were non Hodgkin's lymphoma and acute myeloid leukemia, with $4(23.6 \%)$ occurrences. The most frequent nursing diagnoses were: ineffective protection and risk of infection, with 17 (100\%) occurrences; risk of impaired oral mucosa and risk of falls, with $12(70.6 \%)$ cases. Conclusion: There was a predominance of Nursing diagnoses of ineffective protection and risk of infection, these were related to cancer, chemotherapy, invasive procedures and immunosuppression.
\end{abstract}

Descriptors: Nursing Diagnosis. Hematology. Oncology Nursing. Nursing Process.

\title{
RESUMEN
}

Objetivo: identificar los diagnósticos de Enfermería más frecuentes en pacientes con enfermedades oncohematológicas sometidas a tratamiento quimioterápico antineoplásico. Método: estudio retrospectivo, documental. Los datos fueron recolectados de 17 prontuarios de pacientes hospitalizados en la unidad de oncohematología de un Hospital Público de Enseñanza, en marzo de 2017. Los datos fueron analizados por estadística descriptiva. Resultados: la mayoría de los prontuarios eran de pacientes del sexo masculino $(70,6 \%$ ); el promedio de edad fue de 41,2 años. Los tipos de cáncer más frecuente fueron linfoma no Hodgkin y leucemia mieloide aguda, con 4 (23,6\%) ocurrencias. Los diagnósticos de Enfermería más frecuentes fueron: protección ineficaz y riesgo de infección, con 17 (100\%) ocurrencias; riesgo de mucosa oral perjudicial y riesgo de caídas, con $12(70,6 \%)$ casos. Conclusión: Hubo predominio de los diagnósticos de Enfermería de protección ineficaz y riesgo de infección, estos estuvieron relacionados al cáncer, al tratamiento quimioterápico, a la realización de procedimientos invasivos e inmunosupresión.

Descriptores: Diagnóstico de Enfermería. Hematología. Enfermería Oncológica. Proceso de Enfermería.

\section{INTRODUÇÃO}

Em 2018, as estimativas de incidência das doenças oncohematológicas mostraram índices de aproximadamente 10.800 casos de leucemias, 10.180 casos de linfoma não Hodgkin e 2.530 de linfomas de Hodgkin para ambos os sexos no Brasil. ${ }^{1}$
A quimioterapia antineoplásica representa o tratamento de escolha quando há acometimento do sistema hematopoético e nos casos de neoplasias malignas agudas. ${ }^{2}$ Entretanto, traz uma série de efeitos colaterais, tais como náuseas, vômito, fadiga, dor, anorexia, dispneia, mialgia, febre, alopecia, anemia, neutropenia, resfriado, mucosite, diarreia, 
suscetibilidade a infecções e ansiedade $^{3}$, requerendo habilidades e conhecimento do enfermeiro acerca das especificidades clínicas dos pacientes oncohematológicos e os efeitos colaterais relacionados ao tratamento, visto que os cuidados de Enfermagem devem ser focados na prevenção e no controle das possíveis complicações terapêuticas. $^{4}$

Ao considerar a complexidade em assistir o paciente oncohematológico em tratamento quimioterápico, presume-se a necessidade de o enfermeiro implementar o Processo de Enfermagem (PE) de forma efetiva, utilizando a ferramenta da Prática Baseada em Evidência (PBE), e proporcionar um cuidado individualizado, seguro e integral.

O PE constitui-se em uma metodologia assistencial multifacetada e multidimensional que objetiva organizar, humanizar e qualificar a prática clínica da Enfermagem atribuindo cientificidade, visibilidade, reconhecimento e valorização à profissão e favorecendo a apropriação da autonomia no desenvolvimento do raciocínio clínico e da tomada de decisão. ${ }^{5}$

O conhecimento do perfil clínico dos pacientes assistidos orienta o enfermeiro na compreensão das bases terapêuticas utilizadas, bem como no raciocínio clínico frente ao foco do diagnóstico de Enfermagem (DE). ${ }^{6}$
Entende-se por DE o julgamento clínico acerca das respostas do indivíduo, família ou da comunidade a condições de saúde, processos de vida ou vulnerabilidades. Constitui a base para a seleção das intervenções de Enfermagem, sinalizando o alcance dos resultados esperados, os quais são de responsabilidade do enfermeiro. Sua importância compreende o estabelecimento de uma linguagem padronizada, a qual possibilita uma comunicação clara entre os membros da equipe multiprofissional e o aperfeiçoamento contínuo do PE individualizado (NANDA, 2015). ${ }^{7}$

Estudos sobre a prevalência e a frequência de diagnósticos de Enfermagem e de seus indicadores em populações específicas são fundamentais à prática clínica do enfermeiro, pois possibilitam inferir, de maneira fidedigna, um diagnóstico e permitem estimar as chances de um indivíduo ter ou não um determinado fenômeno. ${ }^{8}$ A identificação do DE com exatidão favorece diretamente a efetividade e a excelência do PE, o que proporciona a segurança do paciente e a melhora da qualidade assistencial.

Nesse sentido, este estudo objetivou identificar os diagnósticos de Enfermagem mais frequentes em pacientes com doenças oncohematológicas submetidos a tratamento quimioterápico antineoplásico. 


\section{MÉTODOS}

Tratou-se de um estudo retrospectivo, documental, de análise de prontuários de pacientes hospitalizados na unidade de oncohematologia de um Hospital Público de Ensino localizado no interior do Sudeste brasileiro. A unidade de oncohematologia supracitada possui seis leitos de internação ativos que atendem à especialidade de oncohematologia e iniciou, recentemente, a realização do transplante autólogo de medula óssea.

Foram incluídos, no estudo, pacientes com doenças oncohematológicas submetidos somente ao tratamento quimioterápico e hospitalizados no período de setembro de 2016 a fevereiro de 2017. Foram excluídos, da pesquisa, pacientes em tratamento radioterápico e submetidos a outros tipos de tratamentos e sem diagnóstico médico de câncer oncohematológico.

A coleta de dados foi realizada em março de 2017 e constou de análise documental de fonte secundária e prontuários de pacientes hospitalizados que atenderam aos critérios de inclusão do estudo. Para a extração de dados, foi elaborado um instrumento estruturado, a partir da literatura pertinente ${ }^{9}$, contendo: (1) dados sociodemográficos; (2) dados clínicos e (3) perfil de títulos diagnósticos de Enfermagem.
As variáveis sociodemográficas analisadas incluíram: sexo, faixa etária, cor, ocupação e estado civil. As variáveis clínicas consideradas foram as seguintes: tipo de câncer, comorbidades, alergia medicamentosa e permanência hospitalar. Para estabelecer o DE, de acordo com a taxonomia II da NANDA Internacional $(\text { NANDA-I })^{7}, \quad$ coletaram-se dados referentes aos fatores de risco ou ao fator relacionado, características definidoras e títulos diagnósticos de Enfermagem documentados nos registros de Enfermagem.

Os dados de interesse levantados nos prontuários foram armazenados em planilha eletrônica do programa Excel® for Windows XP®, validados por dupla digitação, com as codificações das variáveis de estudo e um dicionário (codebook) com a descrição de cada variável. Na sequência, foram exportados para o software Statistical Package for the Social Sciences ${ }^{\circledR}$ (SPSS), versão 20, para processamento e análise. A análise estatística foi realizada por meio da distribuição de frequências absolutas (n) e percentuais (\%) para variáveis categóricas e média e desvio padrão para as variáveis numéricas. Os resultados foram apresentados por meio de tabelas.

Ressalta-se que foram atendidos os aspectos éticos e legais preconizados na resolução 466/2012 do Conselho Nacional 
de Saúde. O projeto foi apreciado e aprovado pelo Comitê de Ética e Pesquisa de uma Universidade Federal sob o parecer número 1.457.721, em março de 2016, com dispensa do Termo de Consentimento Livre e Esclarecido.

\section{RESULTADOS}

Verificou-se um total de 35 prontuários. Destes, $18 \quad(51 \%)$ não atenderam aos critérios de elegibilidade do estudo e foram excluídos, conforme os critérios descritos a seguir: doenças oncohematológicas submetidas a outra modalidade de tratamento $(n=7)$; citopenias a esclarecer (n=2); púrpura trombocitopênica imunológica $(n=2)$; síndrome mielodisplásica $\quad(\mathrm{n}=2)$; adenomegalia a esclarecer ( $n=1)$; câncer de próstata $(\mathrm{n}=1)$; doença hematológica $(\mathrm{n}=1)$; neutropenia febril $(\mathrm{n}=1)$; carcinoma em dorso nasal fora de tratamento $(n=1)$. Os prontuários elegíveis, de acordo com os critérios de inclusão, foram 17 (49\%).

Evidenciou-se que a maioria dos prontuários era de pacientes do sexo masculino $12(70,6 \%)$. A faixa etária variou entre 15 e 68 anos, com média de 41,2 anos e desvio padrão de 18,5. Destaca-se a prevalência da cor branca, com oito $(47,1 \%)$ pacientes. Quanto ao estado civil, a maioria (52,9\%) dos pacientes era solteira. Com relação à ocupação, em cinco $(29,4 \%)$ prontuários não havia esse registro. Os dados referentes ao perfil sociodemográfico foram reunidos na tabela 1 , a seguir.

Tabela 1 - Distribuição das características sociodemográficas identificadas nos prontuários de pacientes oncohematológicos em tratamento quimioterápico. Uberaba, Minas Gerais, Brasil, 2017. $(\mathrm{n}=17)$

\begin{tabular}{l}
\hline \multicolumn{2}{c}{ Perfil Sociodemográfico } \\
\begin{tabular}{|l|c|c}
\hline Sexo & 12 & $\%$ \\
\hline Masculino & 5 & 70,6 \\
\hline Feminino & 5 & 29,4 \\
\hline Faixa Etária & 2 & 29,4 \\
\hline $15-29$ & 4 & 17,6 \\
\hline $30-39$ & 6 & $23,6 \%$ \\
\hline $40-59$ & & $29,4 \%$ \\
\hline $60-68$ & 8 & 47,1 \\
\hline Cor & 6 & 35,3 \\
\hline Branca & 3 & 17,6 \\
\hline Parda & & \\
\hline Preta & 9 & 52,9 \\
\hline Estado Civil & \multicolumn{2}{|c}{} \\
\hline Solteiro & & \\
\hline
\end{tabular}
\end{tabular}




\begin{tabular}{l|l|c}
\hline Casado & 6 & 35,3 \\
\hline União Estável & 1 & 5,9 \\
\hline Sem registro desta variável & 1 & 5,9 \\
\hline Ocupação & 5 & 29,4 \\
\hline Sem registro desta variável & 4 & 23,5 \\
\hline Estudante & 1 & 5,9 \\
\hline Operador de máquinas & 1 & 5,9 \\
\hline Motorista & 1 & 5,9 \\
\hline Serviços gerais em zona rural & 1 & 5,9 \\
\hline Cozinheiro & 1 & 5,9 \\
\hline Pedreiro & 1 & 5,9 \\
\hline Auxiliar de fotografia & 1 & 5,9 \\
\hline Do lar & 1 & 5,9 \\
\hline Comerciante & & \\
\hline
\end{tabular}

Fonte: Elaboração própria, 2017.

Dentre os principais tipos de câncer, destacaram-se o Linfoma não Hodgkin e a Leucemia mieloide aguda, com quatro (23,6\%) ocorrências, respectivamente. Em relação às comorbidades, oito $(47,1 \%)$ pacientes não apresentaram, no entanto, ressalta-se que a hipertensão arterial sistêmica foi a comorbidade associada mais frequente em cinco $(29,4 \%)$ pacientes. Quanto à presença de alergia medicamentosa, $14(82,4 \%)$ pacientes não possuíam (Tabela 2).

Em relação à permanência hospitalar, constatou-se a variação de, no mínimo, quatro a 94 dias de hospitalização, com média de 25,1 dias e desvio padrão de 24,6 dias.

Tabela 2 - Perfil clínico identificado nos prontuários de pacientes oncohematológicos em quimioterapia. Uberaba, Minas Gerais, Brasil, 2017. $(n=17)$

\begin{tabular}{l|c|c}
\hline Perfil Clínico & \multicolumn{2}{c}{$\%$} \\
\hline Tipo de Câncer & 4 & 23,6 \\
\hline Linfoma não Hodgkin & 4 & 23,6 \\
\hline Leucemia mieloide aguda & 2 & 11,8 \\
\hline Leucemia linfoide aguda de células B & 2 & 11,8 \\
\hline Mieloma múltiplo & 1 & 5,9 \\
\hline Leucemia linfoide aguda de células T & 1 & 5,9 \\
\hline $\begin{array}{l}\text { Linfoma de Hodgkin } \\
\text { Linfoma de Burkitt e Leucemia linfoide } \\
\text { aguda de células B }\end{array}$ & 1 & 5,9 \\
\hline $\begin{array}{l}\text { Plasmocitoma mediastinal e mieloma } \\
\text { múltiplo }\end{array}$ & & \\
\hline Comorbidades & 8 & 47,1 \\
\hline Não apresentavam & & \\
\hline
\end{tabular}




\begin{tabular}{l|c|c}
\hline Hipertensão arterial sistêmica & 5 & 29,4 \\
\hline Epilepsia & 2 & 11,8 \\
\hline Trombose venosa profunda & 2 & 11,8 \\
\hline Periodontopatia & 1 & 5,9 \\
\hline Sífilis & 1 & 5,9 \\
\hline Hipotireoidismo & 1 & 5,9 \\
\hline Síndrome da imunodeficiência adquirida & 1 & 5,9 \\
& & \\
\hline Hepatite B crônica & 1 & 5,9 \\
\hline Alergia Medicamentosa & 14 & 82,4 \\
\hline Não & & 5,9 \\
\hline Sim & 1 & 5,9 \\
\hline \multicolumn{1}{c|}{ Dipirona } & 1 & 5,9 \\
\hline \multicolumn{1}{c|}{ Diclofenaco Potássico } & 1 & \\
\hline
\end{tabular}

Fonte: Elaboração própria, 2017.

Foram identificados 30 diagnósticos reais e dez diagnósticos de risco, presentes nos 17 pacientes oncohematológicos, enquanto permaneceram em tratamento quimioterápico antineoplásico.

Os principais DE identificados foram: proteção ineficaz e risco de infecção em 17 casos (100\%); risco de mucosa oral prejudicada e risco de quedas em 12 casos (70,6\%); dor aguda, hipertermia, constipação e nutrição desequilibrada - menor do que as necessidades corporais em 11 casos $(64,7 \%)$ (Tabela 3).
Outros DE, identificados nos prontuários analisados, foram menos frequentes. Dentre eles, destacam-se: volume de líquidos deficiente; mobilidade no leito prejudicada; deambulação prejudicada; risco de desequilíbrio na temperatura corporal; diarreia, com três casos (17,6\%); risco de função hepática prejudicada; confusão aguda; dentição prejudicada; tristeza crônica; intolerância à atividade; risco de perfusão renal ineficaz em dois casos $(11,8 \%)$; desesperança; risco de resposta alérgica; risco de suicídio; hipotermia e baixa autoestima situacional em um caso $(5,9 \%)$.

Tabela 3 - Títulos diagnósticos de Enfermagem, fatores relacionados e fatores de risco identificados nos prontuários de pacientes oncohematológicos em quimioterapia. Uberaba, Minas Gerais, Brasil, 2017. $(\mathrm{n}=17)$

\begin{tabular}{l|c|c}
\hline Títulos Diagnósticos/ Fator Relacionado/ & \multicolumn{2}{c}{ n } \\
Fator de Risco & 17 & $\%$ \\
\hline Proteção Ineficaz & & 100 \\
\hline
\end{tabular}




\begin{tabular}{|c|c|c|}
\hline Câncer & 14 & 82,4 \\
\hline Regime de Tratamento & 2 & 11,8 \\
\hline Câncer/Regime de Tratamento & 1 & 5,9 \\
\hline Risco de Infecção & 17 & 100 \\
\hline Procedimento Invasivo & 10 & 58,8 \\
\hline Imunossupressão & 4 & 23,5 \\
\hline Procedimento Invasivo/Imunossupressão & 3 & 17,6 \\
\hline Risco de Mucosa Oral Prejudicada & 12 & 70,6 \\
\hline Quimioterapia & 12 & 70,6 \\
\hline Risco de Quedas & 12 & 70,6 \\
\hline Outros (Morse moderado a elevado) & 11 & 64,7 \\
\hline Anemia(Morse moderado a elevado) & 1 & 5,9 \\
\hline Dor Aguda & 11 & 64,7 \\
\hline Agente Lesivo Biológico & 11 & 64,7 \\
\hline Hipertermia & 11 & 64,7 \\
\hline Doença & 5 & 29,4 \\
\hline Sepse & 3 & 17,6 \\
\hline Outro (Neutropenia febril) & 2 & 11,8 \\
\hline Doença/Sepse & 1 & 5,9 \\
\hline Constipação & 11 & 64,7 \\
\hline Hábitos de Evacuação Irregulares & 9 & 52,9 \\
\hline Mudança Ambiental Recente & 1 & 5,9 \\
\hline Outro (Fezes ressecadas) & 1 & 5,9 \\
\hline Nutrição Desequilibrada: menor do que as necessidades corporais & 11 & 64,7 \\
\hline Ingestão Alimentar Insuficiente & 10 & 58,8 \\
\hline Incapacidade de Ingerir os Alimentos/Ingestão Alimentar Insuficiente & 1 & 5,9 \\
\hline Náusea & 10 & 58,8 \\
\hline Regime de Tratamento & 10 & 58,8 \\
\hline Mucosa Oral Prejudicada & 9 & 52,9 \\
\hline Imunossupressão & 2 & 11,8 \\
\hline Regime de Tratamento & 7 & 41,2 \\
\hline Ansiedade & 9 & 52,9 \\
\hline Ameaça à Condição Atual & 4 & 23,5 \\
\hline Abuso de Substância & 1 & 5,9 \\
\hline Mudança Importante & 3 & 17,7 \\
\hline Crise Situacional & 1 & 5,9 \\
\hline Volume de Líquidos Excessivo & 9 & 52,9 \\
\hline Mecanismo Regulador Comprometido & 9 & 52,9 \\
\hline Risco de Sangramento & 8 & 47,1 \\
\hline Coagulopatia inerente (Trombocitopenia) & 8 & 47,1 \\
\hline Fadiga & 8 & 47,1 \\
\hline Condição Fisiológica & 8 & 47,1 \\
\hline Integridade da Pele Prejudicada & 7 & 41,2 \\
\hline Fator Mecânico & 1 & 5,9 \\
\hline Imunodeficiência & 1 & 5,9 \\
\hline Outros (Incisão cirúrgica, fissura anal) & 5 & 29,4 \\
\hline Débito Cardíaco Diminuído & 6 & 35,3 \\
\hline Pós-carga alterada & 6 & 35,3 \\
\hline Desobstrução Ineficaz de Vias Aéreas & 6 & 35,3 \\
\hline Secreções Retidas & 5 & 29,4 \\
\hline Muco Excessivo & 1 & 5,9 \\
\hline Deficit no Autocuidado para Banho/Higiene íntima/Vestir-se & 6 & 35,3 \\
\hline Outro (Fadiga) & 3 & 17,6 \\
\hline
\end{tabular}




\begin{tabular}{l|c|c}
\hline Fraqueza & 3 & 17,6 \\
\hline Padrão Respiratório Ineficaz & 5 & 29,4 \\
\hline Outros (Dispneia, taquipneia) & 4 & 23,5 \\
\hline Fadiga da Musculatura Respiratória/Dispneia & 1 & 5,9 \\
\hline Padrão de Sono Prejudicado & 5 & 29,4 \\
\hline Barreira Ambiental & 5 & 29,4 \\
\hline Ventilação Espontânea Prejudicada & 4 & 23,5 \\
\hline Fadiga da Musculatura Respiratória & 4 & 23,5 \\
\hline Risco de Glicemia Instável & 4 & 23,5 \\
\hline Condição de Saúde Física Comprometida & 4 & 23,5 \\
\hline
\end{tabular}

Fonte: Elaboração própria, 2017.

\section{DISCUSSÃO}

A partir da análise documental dos 17 prontuários de pacientes hospitalizados na unidade de oncohematologia, observouse que o sexo masculino $(70,6 \%)$ se destaca sobre o feminino $(29,4 \%)$. Além disso, verificou-se a predominância dos tipos de cânceres Leucemia mieloide aguda e Linfoma não Hodgkin, e a maioria dos pacientes não apresentou comorbidades associadas ou alergia medicamentosa.

Segundo estimativas do INCA, para o Brasil, serão 5.940 casos novos de leucemia em homens e 4.860 em mulheres para cada ano do biênio 2018-2019. Portanto, esses valores correspondem a um risco estimado de cinco casos novos a cada 100 mil homens e quatro casos novos para cada 100 mil mulheres. O INCA descreve a maior incidência de Linfoma não Hodgkin para o sexo masculino (5.370 casos) em comparação ao sexo feminino (4.810 casos). ${ }^{1}$ Diante disso, observa-se que o sexo masculino apresenta um potencial representativo para o desenvolvimento de um tipo de câncer oncohematológico.

Foram identificados 30 diagnósticos de Enfermagem reais e dez diagnósticos de risco. Dentre esses, os mais frequentes foram: proteção ineficaz; risco de infecção; risco de mucosa oral prejudicada; risco de quedas; dor aguda; hipertermia; constipação e nutrição desequilibrada: menor do que as necessidades corporais.

Outros

estudos

também

evidenciaram a ocorrência destes diagnósticos em pacientes oncohematológicos, destacando a proteção ineficaz, o risco de infecção, mucosa oral prejudicada, hipertermia, risco de sangramento, fadiga, dor aguda e nutrição desequilibrada: menor do que as necessidades diárias. ${ }^{9}$ Um estudo de revisão integrativa identificou os diagnósticos de Enfermagem segundo a NANDA-I entre pacientes hospitalizados com câncer e evidenciou os mais comuns: ansiedade, conhecimento deficiente, constipação, deficit de autocuidado para 
banho/higiene, distúrbio da imagem corporal, dor aguda e crônica, medo, padrão de sono alterado, risco de infecção e risco de volume de líquidos deficiente. ${ }^{10}$ Os diagnósticos de Enfermagem identificados neste estudo e recomendados pela literatura científica ${ }^{9,10}$, para pacientes com doença oncohematológica, corroboram com os resultados alcançados. A utilização de uma taxonomia padronizada fornece subsídios para a Enfermagem Baseada em Evidências (EBE) e a uniformização do cuidado de pacientes oncohematológicos.

O diagnóstico de proteção ineficaz é definido como a diminuição na capacidade de proteger-se de ameaças internas ou externas como doenças ou lesões. ${ }^{7}$ Neste estudo, esteve relacionado ao diagnóstico de câncer e ao regime de tratamento, especificamente, a quimioterapia antineoplásica.

A utilização de medicamentos antineoplásicos causa neutropenia e aumenta o risco de sepse. ${ }^{11}$ A neutropenia febril é uma consequência potencialmente fatal do efeito mielossupressor da quimioterapia que leva à hospitalização, à administração de antibióticos por via endovenosa, a reduções de dose e aos atrasos de tratamento, os quais impactam a qualidade de vida e o resultado da sobrevida global dos pacientes. ${ }^{12}$
O diagnóstico de Enfermagem risco de infecção esteve associado à realização de procedimentos invasivos. Dentre esses, destacam-se: a punção de cateter totalmente implantado que, diante da complexidade relacionada ao seu manuseio, é imprescindível à implementação de intervenções de Enfermagem que visem à punção segura com a utilização de técnica asséptica; a manutenção e a troca do curativo e a manutenção do sistema fechado para a prevenção de infecção. ${ }^{13}$ Essas ações objetivam a prevenção de eventos adversos com vistas a promover uma assistência segura para o paciente oncológico.

Em relação ao diagnóstico de risco de mucosa oral prejudicada, este apresenta, como principal complicação, a mucosite, a qual é frequentemente observada em pacientes submetidos à quimioterapia e consiste em lesões ulcerativas da via oral e/ou gastrointestinal, resultando em dor, dificuldade em comer, falar e dormir, impactando diretamente a qualidade de vida. ${ }^{14}$ As intervenções de Enfermagem, frente ao diagnóstico real e de risco de mucosa oral prejudicada, são indispensáveis e devem ser implementadas a fim de prevenir a evolução da lesão, pois este diagnóstico pode apresentar implicações negativas relacionadas a outros DE como risco de infecção, dor 
aguda e nutrição desequilibrada: menor do que as necessidades corporais. ${ }^{9}$

$\mathrm{O}$ risco de quedas foi identificado a partir de registro diário dos enfermeiros durante a hospitalização do paciente. Estima-se que um em cada cinco pacientes com câncer desenvolva um novo fator de risco para quedas na vigência do tratamento quimioterápico sendo que, em idosos, o risco é $17 \%$ maior se comparado a adultos jovens. $^{15}$ A quimioterapia potencializa o risco de quedas no paciente oncohematológico e, diante disso, se faz necessária a implementação de medidas para a sua prevenção.

A dor relacionada ao câncer frequentemente gera consequências negativas para os pacientes oncológicos que interfere em suas atividades de vida diária, estado emocional, nível de atividade, qualidade do sono e nutrição. Além disso, vale destacar que eles demonstram conhecimento e atitude inadequados em relação ao uso de analgésicos. ${ }^{16}$

Nesse cenário, intervenções educacionais, dirigidas aos pacientes, podem reduzir a intensidade da dor oncológica por meio de, pelo menos, um dos sete componentes principais: melhorar o conhecimento sobre a natureza da dor relacionada ao câncer; ajudar na comunicação sobre a dor; melhorar a avaliação da dor; melhorar a prescrição de analgésicos; abordar barreiras à não adesão analgésica; sensibilizar para estratégias não farmacológicas de gestão da dor e promover a reavaliação. O manejo efetivo da dor oncológica resulta em aumento da qualidade de vida para pacientes com câncer e diminuição do período de hospitalização. $^{17}$

O diagnóstico de constipação é comumente apresentado por pacientes oncológicos. Se a constipação não for tratada de maneira adequada, os pacientes podem sofrer consequências negativas tais como anorexia, náuseas, impactação intestinal ou perfuração intestinal. Dessa maneira, sua prevenção e tratamento consistem em um componente essencial da prática oncológica de Enfermagem e devem incluir intervenções baseadas em evidência. $^{18}$

Requer a atenção do enfermeiro o diagnóstico de Enfermagem nutrição desequilibrada: menor do que as necessidades corporais. É de suma importância que a equipe de Enfermagem se empenhe no estímulo da ingesta oral do paciente, orientação quanto à preparação dos alimentos e ao reforço da necessidade de alimentação para a sua recuperação. ${ }^{9}$

Durante os ciclos de quimioterapia, os pacientes apresentam rejeição significativa de alimentos. Refeições completas, como o almoço e o jantar, são menos toleradas pelos pacientes, no 
entanto, as refeições mais rápidas, como os lanches, possuem melhor aceitação. Nesse sentido, uma grande variedade de alimentos pode ser oferecida aos pacientes para melhorar sua nutrição durante o tratamento quimioterápico antineoplásico. ${ }^{19}$

Os efeitos colaterais do tratamento, como náuseas, vômitos, diarreia, mucosite, constipação e perda de apetite, contribuem para a baixa aceitação da dieta, impactam o estado geral do paciente e sinalizam para o estabelecimento do diagnóstico de nutrição desequilibrada: menor do que as necessidades corporais. ${ }^{20}$

A identificação dos diagnósticos de Enfermagem evidenciados neste estudo contribui para o planejamento da assistência e a execução do PE na prática clínica do enfermeiro, uma vez que orienta o raciocínio diagnóstico e a consequente tomada de decisão acerca do plano de cuidados. A assistência de Enfermagem oncohematológica qualificada, baseada em evidências, proporciona intervenções mais eficazes, a promoção de um cuidado humanizado, a segurança e satisfação do paciente.

Considera-se a relevância da apropriação do PE, enquanto ferramenta de qualidade assistencial e sistematização da assistência de Enfermagem nas unidades de oncohematologia, uma vez que a complexidade e a magnitude da doença oncohematológica exigem, dos enfermeiros, instrumentos cada vez mais eficazes para garantir a qualidade do cuidado em saúde.

\section{CONCLUSÃO}

Os diagnósticos de Enfermagem mais frequentes foram: proteção ineficaz; risco de infecção; risco de mucosa oral prejudicada; risco de quedas; dor aguda; hipertermia; constipação e nutrição desequilibrada: menos do que as necessidades corporais. Tais diagnósticos estiveram relacionados ao próprio câncer, à realização de procedimentos invasivos, ao tratamento quimioterápico, ao risco de quedas identificado a partir de registro diário da avaliação dos enfermeiros, a hábitos de evacuação irregulares e à ingestão alimentar insuficiente, caracterizada pela rejeição aos alimentos.

Destaca-se a importância de sumarizar os diagnósticos de Enfermagem frequentes em pacientes oncohematológicos, pois eles orientam o enfermeiro quanto ao raciocínio clínico/diagnóstico e fundamentam a execução do processo de Enfermagem individualizado.

Como limitações do estudo, observou-se um reduzido tamanho amostral de pacientes submetidos à quimioterapia no período de coleta dos dados. Destaca-se que, nos prontuários 
analisados, foram encontradas incompletudes quanto às características sociodemográficas como a ausência de informação quanto à ocupação e ao estado civil.

\section{REFERÊNCIAS}

1. Instituto Nacional de Câncer José Alencar Gomes da Silva. Estimativa 2018: incidência de câncer no Brasil [Internet]. Rio de Janeiro: INCA; 2018 [citado em 20 maio 2018]. Disponível em: http://www1.inca.gov.br/estimativa/2018/e stimativa-2018.pdf

2. Ministério da Saúde (Brasil). Manual de bases técnicas da oncologia - SIA/SUS sistema de informações ambulatoriais [Internet]. 2ed. Brasília, DF: Ministério da Saúde; 2016 [citado em 10 ago 2017]. Disponível em:

http://biblioteca.cofen.gov.br/wpcontent/uploads/2016/07/manual_de_bases _tecnicas_oncologia.pdf

3. Gozzo TO, Souza SG, Moysés AMB, Carvalho RAO, Ferreira SMA.

Conhecimento da equipe de enfermagem acerca de eventos adversos do tratamento quimioterápico. Ciênc Cuid Saúde [Internet]. 2015 [citado em 20 ago 2017]; 14(2):1058-66. Disponível em: http://periodicos.uem.br/ojs/index.php/Cie ncCuidSaude/article/view/25040/pdf_363 4. Carlucci VDS, Braga FTMM, Rei ED, Silveira RCCP. Cuidados de enfermagem a pacientes onco-hematológicos submetidos a altas doses de quimioterapia: revisão integrativa. Rev Enferm UFPE On Line [Internet]. 2016 [citado em 20 ago 2017]; 10(3):1544-55. Disponível em: https://periodicos.ufpe.br/revistas/revistaen fermagem/article/view/11096/12553

5. Souza Júnior DI, Ribeiro JHM, Santos RP, Fagundes KVDL, Dias PF, Mendes MA. Impasses, condições e potencialidades à implementação do processo de enfermagem na prática hospitalar brasileira: revisão integrativa. Rev Enferm UFPE On Line [Internet]. 2017 [citado em 23 ago 2017]; 11(2):65666. Disponível em:

https://periodicos.ufpe.br/revistas/revistaen fermagem/article/view/11985/14544 6. Silva MM, Silva JA, Esteves LO, Mesquita MGR, Stipp MAC, Duarte SMC. Perfil sociodemográfico e clínico de pessoas em tratamento quimioterápico: subsídios para o gerenciamento em enfermagem. Rev Eletrônica Enferm. [Internet]. 2013 [citado em 23 ago 2017]; 15(3):704-12. Disponível em: https://www.fen.ufg.br/revista/v15/n3/pdf/ v15n3a12.pdf

7. Herdman TH, Kamitsuru S. Nanda Internacional. Diagnósticos de Enfermagem da NANDA-I: definições e classificação 2015-2017. 10ed. Porto Alegre: Artmed; 2015.

8. Fernandes MIC, Medeiros ABA, Macedo BM, Vitorino ABF, Lopes MVO, Lira ALBC. Prevalência do diagnóstico de enfermagem volume de líquidos excessivo em pacientes submetidos à hemodiálise. Rev Esc Enferm USP. [Internet]. 2014 [citado em 23 ago 2017]; 48(3):446-53. Disponível em: http://www.scielo.br/pdf/reeusp/v48n3/pt 0080-6234-reeusp-48-03-446.pdf

9. Sousa RM, Santo FHE, Santana RF, Lopes MVO. Diagnósticos de enfermagem identificados em pacientes oncohematólogicos: mapeamento cruzado. Esc Anna Nery Rev Enferm. [Internet]. 2015 [citado em 23 ago 2017]; 19(1):54-65. Disponível em: http://www.scielo.br/pdf/ean/v19n1/14148145-ean-19-01-0054.pdf 10. Jomar RT, Bispo VRS. The most common nursing diagnosis among adults/seniors hospitalized with cancer: integrative review. Ecancer Med Sci. [Internet]. 2014 [citado em 30 ago 2017]; 8(462). Disponível em: https://ecancer.org/journal/8/full/462-themost-common-nursing-diagnosis-amongadults-seniors-hospitalised-with-cancer- 
integrative-review.php. doi:

10.3332/ecancer.2014.462

11. Vioral AN, Wentley D. Managing

oncology neutropenia and sepsis in the intensive care unit. Crit Care nurs Q.

[Internet]. 2015 [citado em 30 ago 2017];

38(2):165-74. doi:

10.1097/CNQ.0000000000000058

12. O'Brien C, Dempsey O, Kennedy MJ.

Febrile neutropenia risk assessment tool:

Improving clinical outcomes for oncology

patients. Eur J Oncol Nurs. [Internet]. 2014

[citado em 30 ago 2017]; 18(2):167-74.

Disponílve em:

https://www.ejoncologynursing.com/article

/S1462-3889(13)00154-3/pdf. doi:

10.1016/j.ejon.2013.11.002

13. Oliveira TF, Rodrigues MCS.

Enfermagem na prevenção de infecção em cateter totalmente implantado no paciente oncológico. Cogitare Enferm. [Internet]. 2016 [citado em 20 set 2017]; 21(2):01-05. Disponível em:

http://revistas.ufpr.br/cogitare/article/view/ 45523/28556

14. Spolarich AE. Risk management

strategies for reducing oral adverse drug

events. J Evid Based Dent Pract. [Internet].

2014 [citado em 20 set 2017];

14(Suppl):87-94. Disponílve em:

https://www.sciencedirect.com/science/arti cle/pii/S1532338214000736?via\%3Dihub.

doi: 10.1016/j.jebdp.2014.04.009

15. Wildes TM, Dua P, Fowler SA, Miller JP, Carpenter CR, Avidan MS, et al.

Systematic review of falls in older adults with cancer. J Geriatr Oncol. [Internet].

2015 [citado em 30 set 2017]; 6(1):70-83.

Disponível em:

https://www.ncbi.nlm.nih.gov/pmc/articles

/PMC4297689/. doi:

10.1016/j.jgo.2014.10.003

16. Ovayolu N, Ovayolu Ö, Serçe S, Tuna

D, Pirbudak Çöçelli L, Sevinç A. Pain and

quality of life in Turkish cancer patients.

Nurs Health Sci. [Internet]. 2013 [citado

em 30 ago 2017]; 15(4):437-43.

Disponível em:

https://onlinelibrary.wiley.com/doi/epdf/10

$.1111 /$ nhs.12047. doi: 10.1111/nhs.12047
17. Adam R, Bond C, Murchie P.

Educational interventions for cancer pain: a systematic review of systematic reviews with nested narrative review of randomized controlled trials. Patient Educ Couns.

[Internet]. 2015 [citado em 30 ago 2017]; 98(3):269-82. Disponível em: https://www.sciencedirect.com/science/arti cle/pii/S0738399114004765?via\%3Dihub. doi: 10.1016/j.pec.2014.11.003

18. Zhe H. The assessment and management of constipation among patients with advanced cancer in a palliative care ward in China: a best practice implementation project. JBI Database System Rev Implement Rep. [Internet]. 2016 [citado em 30 ago 2017]; 14(5):295-309. doi: 10.11124/JBISRIR2016-002631

19. Prockmann S, Freitas AHR, Ferreira MG, Vieira FGK, Salles RK. Evaluation of diet acceptance by patients with haematological cancer during chemotherapeutic treatment. Nutr Hosp. [Internet]. 2015 [citado em 25 out 2017]; 32(2): 779-84. Disponível em:

http://www.aulamedica.es/nh/pdf/8958.pdf 20. Andrade V, Sawada NO, Barichello E. Qualidade de vida de pacientes com câncer hematológico em tratamento quimioterápico. Rev Esc Enferm USP. [Internet]. 2013 [citado em 25 out 2017]; 47(2):355-61. Disponível em: http://www.scielo.br/pdf/reeusp/v47n2/12. pdf

RECEBIDO: 08/08/2018

APROVADO: 06/12/2018

PUBLICADO: $12 / 2018$ 